\title{
Ubiquitine, cycle cellulaire et réparation de I'ADN
}

" Mais à quoi sert donc l'ubiquitine? " demandions-nous dans un récent numéro de médecine/sciences ( ${ }^{\circ} 5$, vol. 2, p. 282), et les réponses apportées étaient multiples. L'ubiquitine est d'abord impliquée dans la dégradation protéolytique des protéines altérées auxquelles elle se fixe de manière covalente au terme d'un processus complexe, comportant trois étapes : la fixation de l'ubiquitine sur une enzyme activatrice $E_{1}$, puis le transfert de l'ubiquitine activée de $\mathrm{E}_{1}$ à l'enzyme de conjugaison $\mathrm{E}_{2}$, et enfin la liaison à la protéine altérée, qui requiert parfois une troisième enzyme $\mathrm{E}_{3}$. La fixation de l'ubiquitine à certaines protéines de la chromatine et à des récepteurs membranaires $\left(\mathrm{m} / \mathrm{s} n^{\circ} 5\right.$, vol. 2, p. 282) suggérait d'autres rôles pour cette protéine dont l'extraordinaire conservation entre les espèces (seulement trois acides aminés sur 76 different entre la levure et l'homme) indique qu'elle remplit des fonctions essentielles pour les cellules vivantes.

L'une des fonctions pourrait intéresser le cycle cellulaire et la réparation de l'ADN. En effet, S. Jentsch et al., du laboratoire d'A. Varshavsky au MIT (Cambridge, Ma USA), viennent de démontrer qu'un gène de levure (RAD6) dont la mutation a des effets multiples sur la méïose, la stabilité de l'ADN, la fréquence des mutations et le cycle cellulaire; code pour une enzyme de type $\mathrm{E}_{2}[1,2]$. Le produit du gène $R A D 6$ est capable de transférer une molécule d'ubiquitine sur l'histone $\mathrm{H}-2 \mathrm{~B}$. On peut émettre l'hypothèse que l'ubiquitination de protéines de la chromatine change sa conformation, et donc l'accessibilité de l'ADN sousjacent à des protéines diverses. Il se pourrait soit que les propriétés des histones ubiquitinées fussent modifiées, soit que leur ubiquitination conduisît à leur dégradation protéolytique, le résultat étant dans tous les cas un relâchement de la structure chromatinienne.

$\mathrm{Si}$ les réactions d'ubiquitination sont stimulées par la présence de lésions de l'ADN, les changements induits au niveau de la chromatine pourraient permettre l'accès à ces lésions des enzymes de réparation. Des arguments expérimentaux importants existent pour impliquer aussi l'ubiquitine dans le cycle cellulaire des cellules de mammifère [1], et la conservation de cette molécule rend bien probable, au niveau de ces cellules, son intervention dans les phénomènes de réparation de l'ADN. Il y a fort à parier que, d'ores et déjà, de nombreux laboratoires à travers le monde testent l'intervention possible de l'ubiquitine dans les multiples syndromes, les réparatoses, qui comportent une fragilité du matériel génétique et une prédisposition au cancer $\left([3,4]\right.$ et bréve $\mathrm{m} / \mathrm{s} n^{\circ} 5$, vol. 3 , p. 304$)$.

A. $\mathbf{K}$. 1. Sedgwick S, Johnston L. Ubiquitous cycles
of repair. Nature 1987; $329: 109-$ ?.

2. Jentsch S, McGrath JP, Varshavsky A. The yeast DNA repair gene RAD6 encodes a ubiquitin-conjugating enzyme. Nature 1987, 329 ; $131-5$.

3. Aurias A. Aspects cytogénétiques de l'ataxie-télangiectasie. médecire/sciences $1986 ; 6$ : 298-303.

4. Moustacchi E. L'anémie de Fanconi aspects génétiques et moléculaires. médecine/sciences $1987 ; 10$ : 608-12.

\section{BRÈVES}

- Le cerveau a sa propre production d'hormones stéroïdes ces cellules gliales dont le rôle connu jusqu'à présent était la myélinisation des axones dans le système nerveux central. La production de " neurostéroïdes " était suspectée en raison de la présence de prégnénolone et de déhydroépiandrostérone dans le cerveau après ablation des glandes endocrines, gonades et glandes surrénales, donc après élimination des sources périphériques éventuelles. On n'avait pourtant pas défini cette source cérébrale d'hormones stéroïdes. Voilà qui est chose faite immunocytochimiquement à l'aide d'anticorps reconnaissant le cytochrome P-450 scc, enzyme de synthèse des deux hormones à partir du cholestérol. L'enzyme est localisée essentiellement dans la substance blanche, et plus particulièrement dans les mitochondries des oligodendrocytes. On peut donc dire qu'il s'agit pour l'essentiel de "gliostéroïdes ", les premiers à être identifiés. Quelques rares neurones présentent également une immunoréactivité dans le bulbe olfactif et dans les cortex cingulaire et entorhinal. Les oligodendrocytes libèrent sans aucun doute les hormones de façon paracrine et les cibles neurones ou autres cellules gliales ? - peuvent être très éloignées de la source. La visualisation de ces cibles des "gliohormones", c'est-à-dire des cellules porteuses des récepteurs spécifiques, est la prochaine étape de ce puzzle, celle qui permettra peut-être de comprendre leur fonction dans le système nerveux central.

[Le Goascogue C, et al. Science 1987 ; 237 : 1212-4.] à partir des oligodendrocytes,
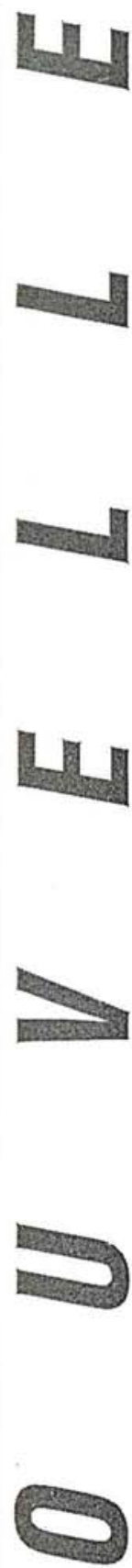

$\mathrm{m} / \mathrm{s} n^{\circ} 1$ vol. 4, janvier 88 
La protéine codée par l'oncogène cellulaire $c$-fos joue peut-être un rôle dans la plasticité du système nerveux central. Jusqu'à présent, on avait montré grâce à des études in vitro que l'expression de l'oncogène cellulaire $c$-fos était régulée de façon rapide et transitoire dans les cellules PC 12 de phéochromocytome de rat par certains facteurs de croissance tels le NGF. Plus récemment, il avait été également démontré sur le même matériel que l'expression du gène était contrôlée par des agents modifiant l'activité des canaux calcium "voltage-dépendants". Deux expériences rapportées indépendamment cet été font état d'une régulation de l'expression de l'oncogène in vivo lors de crises d'épilepsie induites chez le rat par l'injection de métrazole ou par stimulation électrique de la région dorsale de l'hippocampe (phénomène dit de kindling). Le déclenchement des crises d'épilepsie s'accompagne, dans certaines populations de neurones, d'une nette augmentation de la quantité d'ARN messagers et, secondairement, d'une production considérablement accrue de la protéine. L'effet des crises semble s'exercer spécifiquement sur l'expression de certains gènes puisque, par exemple, l'expression de c-ras n'est pas modifiée. Spécifique également la localisation de l'effet des crises dans le cerveau, puisque l'augmentation de l'expression de l'oncogène n'est, dans un premier temps, observée que dans certaines populations de neurones (gyrus dentatus de l'hippocampe, cortex pyriforme et cingulaire) dont l'implication dans les crises d'épilepsie expérimentales étudiées est bien connue. L'expression de l'oncogène est donc manifestement sous la dépendance de mécanismes activant les cellules in vivo. Les auteurs interprètent leurs résultats en suggérant que la protéine fos pourrait être impliquée
" mémoire " au niveau cellulaire. On sait que l'activité de certains neurones, ceux de l'hippocampe par exemple, présente une " mémoire ", c'est-à-dire qu'elle n'est pas identique en réponse à une même stimulation mais au contraire differe en fonction des activations subies précédemment par la cellule. Cette "mémoire cellulaire " devait s'appuyer sur des altérations structurales nécessitant une expression génétique. L'oncogène $c$-fos est aujourd'hui le premier candidat à ce rôle.

[Morgan JI, et al. Science 237 ; 1987 : 192-6.]

[Dragunow M, Robertson HA. Nature 1987 ; 329 : 441-2.]

- Les anticorps antinoyaux de certains malades ayant une atteinte rhumatismale sont dirigés contre la poly-ADPribose polymérase. Cette enzyme nucléaire dont le substrat est le $\mathrm{NAD}^{+}$(nicotinamide dinucléotide phosphate, coenzyme des déshydrogenases), catalyse la fixation de longues chaînes de poly ADPribose sur des protéines chromatiniennes ; cette réaction, stimulée fortement par la présence de cassures de l'ADN, joue très probablement un rôle important dans les phénomènes de réparation de l'ADN. Certains travaux ont suggéré qu'une activation excessive de l'enzyme dans des lymphocytes soumis à une agression par des radicaux oxydants, par exemple libérés au cours d'une réaction inflammatoire, pouvait perturber la réponse immune, peut-être via une déplétion en nucléotides. Chez six malades atteints de différents types de maladies rhumatismales (dont deux syndromes de Sjögren), les auto-anticorps antinoyaux ont été identifiés comme des anticorps antipoly ADP-ribose polymérase.

[Yamanaka $\mathrm{H}$, et al. J Clin Invest $1987 ; 80$ : 900-4.]
- Les protéines plasmatiques qui dépendent de la vitamine $K$ forment des hétérodimères. Ces protéines (prothrombine, facteurs VII, IX, $\mathrm{X}$ et protéines $\mathrm{C}, \mathrm{S}$ et $\mathrm{Z}$ ) contiennent toutes un module N-terminal d'environ 50 acides aminés présentant un haut degré d'homologie. C'est cette portion qui porte les acides $\gamma$-carboxyglutamiques qui lui confèrent une affinité élevée pour les ions $\mathrm{Ca}^{++}$. Cette région comporte aussi une zone hydrophobe qui peut s'apparier avec une chaîne similaire pour former un dimère ; l'homodimère de prothrombine a été détecté en solution. $\mathrm{Si}$ on mélange le fragment de prothrombine (acides aminés I-156, plus facile à manipuler mais doté des mêmes propriétés que la molécule entière) et du facteur $\mathrm{X}$ en présence $\mathrm{d}^{\prime}$ ions $\mathrm{Ca}^{++}$ on peut mettre en évidence la formation d'un hétérodimère entre ces deux protéines, alors que le facteur seul ne se dimérise pas. On a pu également démontrer la formation d'un complexe équimoléculaire entre les protéines $C$ et S. Du fait de l'homologie, toutes les combinaisons sont théoriquement possibles, mais il semble que certaines d'entre elles soient formées préférentiellement ; elles jouent peut-être un rôle important dans la cascade de la coagulation. Ces combinaisons sont données cidessous, la lettre " a " indiquant que le facteur est sous la forme activée.

Xa-prothrombine ; IXa-X ; VIIa-X ; VIIa-IX ; Ca-S

[Harlos K, et al. Nature 1987 ; $330: 82-4$.]

Le danger d'être trop aimé ! Les aquariophiles connaissent bien les "gupies", ces poissons d'eau douce américains dont la femelle est de couleur uniforme et est dépourvue de cette splendide nageoire caudale qui fait la fierté des mâles aux couleurs vives... ainsi que celle de leur 
propriétaire. Les femelles aiment cette "magnificence "... et s'accouplent en priorité avec les mâles les plus colorés, ce qui crée un facteur de sélection en faveur de ce caractère génétique. Il devrait s'ensuivre un " embellissement " continu des mâles au cours de l'évolution, les femelles préférant toujours les plus éclatants d'entre eux. Hélas... les prédateurs détectent, eux aussi, avec prédilection les individus aux couleurs les plus vives... et les mangent, ce qui constitue une autolimitation à la tendance qu'ont les mâles de se parer de coloris toujours plus " chatoyants ". Lorsque les prédateurs deviennent très nombreux, être trop voyant finit par devenir franchement un handicap... et les femelles s'adaptent ! Peut-être lassées de voir leur progéniture issue d'un croisement avec un individu remarquable être la proie préférée des prédateurs, elles se mettent alors à manifester une nette préférence pour l'humilité et la discrétion... et choisissent des mâles plutôt ternes! Éternel conflit entre l'attirance et la recherche de sécurité ? [Bredent F, Stoner G. Nature 1987 ; 329 : 831-3.]

L'antigène prostatespécifique (PSA) est en passe de supplanter la phosphatase acide prostatique (PAP) dans le diagnostic des affections de la prostate. Décrit en 1979, le PSA est formé d'une chaîne de 240 acides aminés ; il a les propriétés d'une protéase à sérine de la famille des kallikréines. Depuis 1980, il a fait l'objet de recherches cliniques, mais l'accord ne semblait pas réalisé sur son intérêt. Un travail américain portant sur 700 sujets devrait permettre d'aboutir à des conclusions précises. Contrairement à la PAP, le PSA est strictement spécifique de la prostate. Son taux normal, obtenu par radioimmunologie, est inférieur à 2,5 nanogrammes par millilitre de plasma. Un simple massage de la $\mathrm{m} / \mathrm{s} n^{\circ} 1$ vol. 4, janvier 88 prostate le double. La demi-vie de la protéine est de 2,2 jours, et l'ablation de l'organe le fait tomber à des niveaux indétectables en deux semaines. Le taux plasmatique du PSA s'élève au cours du cancer de la prostate, en général en proportion du volume de la tumeur. Mais, si le PSA est spécifique d'organe, il n'est pas spécifique de la malignité (pas plus que la PAP d'ailleurs), car son niveau s'élève également dans l'hypertrophie bénigne. Son utilité clinique réside essentiellement dans sa valeur pour le pronostic. Après prostatectomie pour cancer, son taux doit tomber à zéro. Toute activité résiduelle ou réapparition ultérieure du PSA plasmatique signe l'existence d'une extension ou d'une métastase du cancer.

[Stamey TA, et al. $N$ Engl J Med 1987 ; 317 : 909-16.]

Un Un diagnostic par RMN d'une maladie héréditaire du métabolisme. La spectroscopie de résonance magnétique au ${ }^{31} \mathrm{P}$ permet une analyse des métabolites du phosphore obtenue par une méthode non invasive. On peut notamment mesurer la concentration en phosphate minéral (ou inorganique, PI), en esters monophosphorés (PM), diphosphorés, en ATP, ainsi que, dans le muscle, en créatine phosphate. Oberhaensli et al. ont appliqué cette technique à l'étude de l'intolérance au fructose. Dans cette maladie récessive autosomique, le fructose est bloqué au stade de fructose 1-phosphate et ne peut être utilisé. Le diagnostic, important à faire car il est sanctionné par un régime excluant tout apport en fructose direct ou combiné, nécessitait une biopsie hépatique. Le test proposé emploie une charge alimentaire minime en fructose (de l'ordre du gramme, ce qui ne déclenche pas d'hypoglycémie). Elle suffit à provoquer, chez les déficients, des variations importantes de deux paramètres : la concentration en PI s'abaisse de moitié, celle en PM s'élève de $50 \%$. L'épreuve est si sensible que, mise en œuvre après un repas, elle permet de reconnaître si le régime sans fructose est appliqué correctement. Enfin, grâce à elle, il est possible, pour la première fois, de détecter les hétérozygotes : à condition de leur donner la charge habituelle de fructose (50 grammes, bien tolérée par eux) des variations du même ordre sont observées.

[Oberhaensli, RD, et al. Lancet 1987, ii : 931-4.]

口 Bienvenue à l'EGF dans le club des facteurs trophiques agissant dans le système nerveux central! Après le NGF (nerve growth factor), ancêtre des substances trophiques agissant sur le système nerveux périphérique et dont l'activité a été récemment démontrée dans le cerveau, l'EGF (epidermal growth factor) monte, lui aussi, au cerveau. Ce polypeptide mitogène isolé originellement de la glande sous-maxillaire de souris avait été retrouvé récemment dans le système nerveux central. Il existait également des récepteurs spécifiques à l'EGF dans le cerveau mais, jusqu'à présent, son rôle trophique n'avait été mis en évidence que pour des cellules gliales. On sait maintenant, grâce à l'étude de neurones télencéphaliques en culture, que l'EGF favorise leur survie et le développement de neurites. L'activité trophique de l'EGF est comparable à celle d'un autre facteur trophique connu, le FGF (fibroblast growth factor), alors que nombre de substances qui présentent une activité trophique pour d'autres populations cellulaires, essayées dans les mêmes conditions expérimentales, restaient sans effet. L'EGF allonge donc officiellement la liste des facteurs trophiques agissant dans le cerveau, mais celle-ci n'est sûrement pas close car de nombreux prétendants se pressent encore à la porte.

[Morrison RS, et al. Science 238 ; 1987 : 72-5.] 